\title{
Damage Analysis of 3D Frame Structure under Impulsive Load
}

\author{
Wenzhao Fan \& Julin Wang \\ Institute of Building Structures \\ Shanxi Architectural Technical College \\ Taiyuan 030006, China
}

Tel: 86-351-743-2632 E-mail: wangj1_tj@163.com

\author{
Liming Gao \\ Department of Civil Engineering \\ Siegen University \\ Sigen D-57076, Germany \\ E-mail: gaolm_ty@126.com
}

\author{
Shougao Tang \\ School of Aerospace Engineering and Applied Mechanics \\ Tongji University \\ Shanghai 200092, China
}

Tel: 86-21-6513-1712Ｅ-mail: tangshougao@yahoo.com

\begin{abstract}
In this article, we utilized the lumped damage mechanics method to implement the damage analysis to the 3D frame structure under impulsive load. First, take the damage parameters as the interior variable of the system, and based on the finite unit, combine continuum mechanics, fracture mechanics and plastic hinge to analyze the mechanical behaviors of the structure, and finally implement numerical simulation analysis to the frame structure with two layers, and compare the result with the computation result of ABAQUS to validate the feasibility.
\end{abstract}

Keywords: Lumped damage mechanics, Impulsive load, Damage

\section{Introduction}

Since the twin towers of the World Trade Center were stroke by terrorists, the nonlinear analysis and damage computation of structure under sudden impulsive loads are more and more concerned by people. The relative researches can not only establish more reasonable structure design theory, but offer necessary theoretic base for the reliability evaluation and reinforce design of damaged structure.

In recent years, many deep researches about the reaction analysis and damage mechanism of structure under sudden impulsive loads occur and some reasonable methods are put forward one by one (Fabio F, 1991, Xue, 1999, P.334-342, Zhu, 1991, P.21-30, G. Bolzon, 1996, P.1481-1491, Cipollina, A, 1995, P.1113-1126, Marante, 2002, P.1141-1152, Mazza, 1998, Perera, 2000, P.293-302). Based on former researches, we utilize the method of lumped damage mechanics to directly solute the damage of 3D frame structure under the impulsive loads, and implement numerical simulation analysis to one frame structure with two layers, and compare the result with the computation result of ABAQUS to validate the feasibility.

\section{The dynamic equation of 3D structure}

Suppose there are $m$ frame members and $n$ nodes, and the dynamic equation in the time range of $[0, T]$ is

$$
\sum_{e=1}^{m}[B]_{e}^{T}[M]_{e}+\sum_{e=1}^{m}[m]_{e}\{\ddot{U}\}=\{P\} \quad \forall\left\{\dot{U}^{*}\right\}
$$

Where, the matrix $[B]$ is the node displacement function, and the concrete form is 


$$
[B]=\left[\begin{array}{cccccccccccc}
-\frac{m_{1}}{L} & -\frac{m_{2}}{L} & -\frac{m_{3}}{L} & n_{1} & n_{2} & n_{3} & \frac{m_{1}}{L} & \frac{m_{2}}{L} & \frac{m_{3}}{L} & 0 & 0 & 0 \\
-\frac{m_{1}}{L} & -\frac{m_{2}}{L} & -\frac{m_{3}}{L} & 0 & 0 & 0 & \frac{m_{1}}{L} & \frac{m_{2}}{L} & \frac{m_{3}}{L} & n_{1} & n_{2} & n_{3} \\
-t_{1} & -t_{2} & -t_{3} & 0 & 0 & 0 & t_{1} & t_{2} & t_{3} & 0 & 0 & 0 \\
\frac{n_{1}}{L} & \frac{n_{2}}{L} & \frac{n_{3}}{L} & m_{1} & m_{2} & m_{3}-\frac{n_{1}}{L}-\frac{n_{2}}{L}-\frac{n_{3}}{L} & 0 & 0 & 0 \\
\frac{n_{1}}{L} & \frac{n_{2}}{L} & \frac{n_{3}}{L} & 0 & 0 & 0 & -\frac{n_{1}}{L}-\frac{n_{2}}{L}-\frac{n_{3}}{L} & m_{1} & m_{2} & m_{3} \\
0 & 0 & 0 & -t_{1} & -t_{2} & -t_{3} & 0 & 0 & 0 & t_{1} & t_{2} & t_{3} \\
0 & & & & & & & & & & &
\end{array}\right]
$$

Where, $t, m$ and $n$ respectively are the unit vectors of $x, y$ and $z$ axis, and $L$ is the length of the frame member.

$M_{e}^{T}$ is the interior force of the unit e, $M_{e}^{T}=\left(m_{i y}, m_{j y}, N, m_{i z}, m_{j z}, m_{x}\right)$.

Where, $N$ is the axial force, $m_{x}$ is the torque, $m_{i y}$ and $m_{j y}$ are the flexural moments of $i$ and $j$ ports in the face of $x z, m_{i z}$ and $m_{j z}$ are the flexural moments of $i$ and $j$ ports in the face of $x y$ (seen in Figure 1).

$\{U\}^{T}$ is the displacement matrix of the whole structure, $\{U\}^{T}=\left(\{u\}_{1},\{u\}_{2}, \cdots\{u\}_{n}\right)$.

$\{P\}$ is the node exterior force of the structure.

\section{Model of lumped damage mechanics}

\subsection{The damage constitutive equation (Maria, 2003, P.5109-5123)}

For the lumped damage mechanics model, the unit is composed by one elastic girder and two plastic hinges on two ends (seen in Figure 2), and suppose the dissipation of all energies is centralized on the plastic hinges.

Suppose the distortion of the hinge is composed by the plastic distortion and the distortion induced by the damage, i.e.

$$
\left\{\Phi^{h}\right\}=\left\{\Phi^{p}\right\}+\left\{\Phi^{d}\right\}
$$

Where, $\Phi^{p}$ denotes the plastic distortion of the hinge and $\Phi^{d}$ denotes the distortion induced by the damage.

$$
\left\{\Phi^{p}\right\}=\left(\phi_{i y}^{p}, \phi_{j y}^{p}, \delta^{p}, \phi_{i z}^{p}, \phi_{j z}^{p}, \phi_{x}^{p}\right)^{T}
$$

In the frame structure of RC, the plastic corner is mainly induced by the reinforcing steel bar yield, and the fracture of the concrete can be described by following two damage matrixes.

$$
\begin{array}{r}
\left\{D^{+}\right\}=\left(d_{i y}^{+}, d_{j y}^{+}, d_{i z}^{+}, d_{j z}^{+}\right) \\
\left\{D^{-}\right\}=\left(d_{i y}^{-}, d_{j y}^{-}, d_{i z}^{-}, d_{j z}^{-}\right)
\end{array}
$$

Where, + and - respectively denote the damages induced by positive and negative flexural moments (seen in Figure 3 ). The constitutive equation of the plastic hinge is

$$
\left\{\Phi-\Phi^{p}\right\}_{e}=\left[F\left(D^{+}\right)\right]_{e}[M]_{e,+}+\left[F\left(D^{-}\right)\right]_{e,-}[M]_{-}
$$

Where, + and - respectively denote the damages induced by positive and negative flexural moments, $[F(D)]$ denotes the flexibility matrix of the damage unit, and the concrete expressions are

$$
\begin{gathered}
F\left(D^{+}\right)=F^{0}+C\left(D^{+}\right) \\
F\left(D^{-}\right)=F^{0}+C\left(D^{-}\right)
\end{gathered}
$$

In the equation (8) and equation (9), $F^{0}$ denotes the flexibility matrix of the unit in the flexible stage, $C\left(D^{+}\right)$and $C\left(D^{-}\right)$denotes the flexibility matrix induced by the damage of concrete, and the concrete expressions are 


$$
\left[C\left(D^{+}\right)\right]=\left[\begin{array}{cccccc}
\frac{d_{i y}^{+} F_{11}^{0}}{1-d_{i y}^{+}} & 0 & 0 & 0 & 0 & 0 \\
0 & \frac{d_{j y}^{+} F_{22}^{0}}{1-d_{j y}^{+}} & 0 & 0 & 0 & 0 \\
0 & 0 & 0 & 0 & 0 & 0 \\
0 & 0 & 0 & \frac{d_{i z}^{+} F_{44}^{0}}{1-d_{i z}^{+}} & 0 & 0 \\
0 & 0 & 0 & 0 & \frac{d_{j z}^{+} F_{55}^{0}}{1-d_{j z}^{+}} & 0 \\
0 & 0 & 0 & 0 & 0 & 0
\end{array}\right]
$$

3.2 Damage evolvement rate (Marante, 2002, P.1141-1152)

Marante regarded the damage rule of Rankine as the function of the plastic hinge energy release rate, and Figure 4 explains the rule.

From the figure, we can see that the undamaged is confirmed by the following equations.

$$
\begin{array}{lll}
G_{i y}^{+}=R_{i y}^{+}\left(d_{i y}^{+} ; N, m_{x}\right) ; & G_{i y}^{-}=R_{i y}^{-}\left(d_{i y}^{-} ; N, m_{x}\right) \\
G_{i z}^{+}=R_{i z}^{+}\left(d_{i z}^{+} ; N, m_{x}\right) ; & G_{i z}^{-}=R_{i z}^{-}\left(d_{i z}^{-} ; N, m_{x}\right)
\end{array}
$$

The function of $R()$ is used to describe the anti-cracking ability of the plastic hinge $i$, and it is related with the axial force, torque and damage parameters, and it can be confirmed in the experiment. Cipollina et al obtained the expression of $R()$ according to the experiment.

$$
R=G_{c r}\left(N, m_{x}\right)+q\left(N, m_{x}\right) \frac{\ln (1-d)}{1-d}
$$

Where, $G_{c r}$ and $q$ respectively denote the anti-cracking parameters before and after the crack occurs, and both parameters are related with the axial force and torque, and they are confirmed by the following formulas.

$$
\begin{aligned}
& G_{c r}=\frac{1}{2} F_{0} m_{c r}^{2}\left(N, m_{x}\right) \\
& \begin{aligned}
\frac{F_{0} m_{u}^{2}\left(N, m_{x}\right)}{2}= & \left(1-d_{u}\right)^{2} G_{c r}+q\left(1-d_{u}\right) \ln \left(1-d_{u}\right) \\
& -2\left(1-d_{u}\right) G_{c r}+q\left[\ln \left(1-d_{u}\right)+1\right]=0
\end{aligned}
\end{aligned}
$$

In above two formulas, $m_{c r}$ denotes the flexural moment when the crack occurs, $m_{u}$ denotes the maximum flexural moment, and $d_{u}$ denotes the maximum damage value.

\subsection{Yield function}

The effective flexural moments of the plastic hinge, $\bar{m}_{i y}$ and $\bar{m}_{i z}$ are 


$$
\begin{aligned}
& \bar{m}_{i y}= \begin{cases}\frac{m_{i y}}{1-d_{i y}^{+}} & d_{i y}^{+}>0 \\
\frac{m_{i y}}{1-d_{i y}^{-}} & d_{i y}^{-}>0\end{cases} \\
& \bar{m}_{i z}= \begin{cases}\frac{m_{i z}}{1-d_{i z}^{+}} & d_{i z}^{+}>0 \\
\frac{m_{i z}}{1-d_{i z}^{-}} & d_{i z}^{-}>0\end{cases}
\end{aligned}
$$

Replace the actual flexural moment by the effective flexural moment, and the yield function of the plastic hinge $i$ is

$$
f_{i}=f_{i}\left(\bar{m}_{i y}, \bar{m}_{i z}, m_{x}, N, \phi_{i y}^{p}, \phi_{i z}^{p}, \delta_{i}^{p}\right)
$$

So the plastic distortion evolvement rate of the plastic hinge is

$$
\begin{array}{rlrl}
\dot{\phi_{i y}^{p}} & =\dot{\lambda}_{i} \frac{\partial f_{i}}{\partial m_{i y}} \quad ; \quad & \dot{\phi}_{i z}^{p} & =\dot{\lambda}_{i} \frac{\partial f_{i}}{\partial m_{i z}} \\
\dot{\phi_{x}^{p}}=\dot{\lambda}_{i} \frac{\partial f_{i}}{\partial m_{x}} \quad ; & \dot{\delta_{i}^{p}}=\dot{\lambda}_{i} \frac{\partial f_{i}}{\partial N}
\end{array}
$$

And the axial plastic distortion evolvement rate and the plastic corner evolvement rate of the unit are

$$
\begin{gathered}
\dot{\delta}_{x}^{p}=\dot{\lambda}_{i} \frac{\partial f_{i}}{\partial N}+\dot{\lambda}_{i} \frac{\partial f_{j}}{\partial N} \\
\dot{\phi}_{x}^{p}=\dot{\lambda}_{i} \frac{\partial f_{i}}{\partial m_{x}}+\dot{\lambda}_{i} \frac{\partial f_{j}}{\partial m_{x}}
\end{gathered}
$$

\section{Numerical computation of the example}

\subsection{Description of the example}

The example is one single span RC frame structure with two layers. The span is $3.5 \mathrm{~m}$, the height of the layer is $2 \mathrm{~m}$, the Poisson ratio is 0.3 , the elastic modules is $2.633 \mathrm{GPa}$ and the density is $2.5 \mathrm{t} / \mathrm{m}^{3}$. When the pole section is $0.3 \times 0.3 \mathrm{~m}$, the reinforcement assembly is $4 \Phi 10$, and when the girder section is $0.4 \times 0.4 \mathrm{~m}$, the reinforcement assembly is $4 \Phi 12$. The top suffers the impulsive load, which is seen in Figure 5.

In $1 \mathrm{~ms}$, the pressure will increase from zero to the max. $700 \mathrm{KN}$, and then the pressure will keep at the constant in $9 \mathrm{~ms}$, and in the later $10 \mathrm{~ms}$, the pressure will reduce to zero. And the pressure will keep at zero in later analysis (seen in Figure 6).

\subsection{Numerical analysis (Anil K. Chopra, 2005)}

The time interval $[0, T]$ is dispersed into a series of discontinuous time $\left(0, t_{1}, t_{2} \cdots, T\right)$, and we use the finite difference method and the Newmark method to denote the node displacement acceleration by the node displacement, and structure can be analyzed in $[0, T]$. In the appointed time $t_{s}$, the equation (1) can be wrote as

$$
\{L(U)\}=\sum_{e=1}^{m}[B]_{e}^{T}[M]_{e}+\sum_{e=1}^{m}[m]_{e}\{\ddot{U}\}-\{P\}=0
$$

To obtain the displacement at the time $t_{s}$, we can solve it by the following equation.

$$
\{L(U)\} \cong\left\{L\left(U_{0}\right)\right\}+\left[\frac{\partial L}{\partial U}\right]_{\left\{U \in=\left\{U_{0}\right\}\right.}\left\{U-U_{0}\right\}=0
$$

Where, $\left\{U_{0}\right\}$ denotes the displace matrix obtained by the computation before the time $t_{s}$, and $\{U\}$ denotes the displace matrix of the time $t_{s}$ which we want to beg.

We utilize the center difference method to implement explicit time integral to the above motion equation, and offer the 
acceleration which can fulfill the dynamical balance condition when the increment step begins. To obtain the acceleration, we explicitly deduce the speed and displacement on time. The computation of the unit includes confirming the constitutive relation between unit strain and applied materials and the unit stress, and further computing the interior force and obtaining the damage value of the unit finally. The concrete approaches include

(1) According to the strain rate $\mathcal{E}$, compute the unit strain increment $d \varepsilon$.

(2) According to the constitutive relation, compute the stress $\sigma$.

$$
\sigma_{(t+\Delta t)}=f\left(\sigma_{(t)}, d \varepsilon\right)
$$

(3) Integrating node internal force $I_{(t+\Delta t)}$.

(4) Computing the damage value of the unit.

\subsection{Parameter setting}

Take the time increment as $3.0 \times 10^{-5} \mathrm{~s}$. According the design principle of RC, compute and obtain the flexural moment of the girder and pole in various stages.

Pole: $m_{c r}=0.28 \times 10^{5} \mathrm{KN} \mathrm{mm}, m_{u}=1.61 \times 10^{5} \mathrm{KN} \mathrm{mm}$.

Girder: $m_{c r}=0.69 \times 10^{5} \mathrm{KN} \mathrm{mm}, m_{u}=2.53 \times 10^{5} \mathrm{KN} \mathrm{mm}$.

\subsection{Computation and analysis}

Take the sub-module VUMAT of ABAQUS as the interface, adopt Fortran to compile and define the above model of the lumped damage mechanics, and run the program, and the computation result is seen in Figure 7. Use the fixed subprogram EXPLICIT modeling and compute the example, the result is seen in Figure 8.

From Figure 7 and Figure 8, both the method in the article and the commercial software ABAQUS can compute the damage of structure under the impulsive load, but the obvious difference is that the method can compute the damage values of the two ends of the frame member, but ABAQUS can compute the damage value of the whole unit. In fact, the place that damage easily occurs in RC is the joint among frame members, i.e. the end part of the frame members. So, the method in the article more accords with the requirement of the actual engineering.

For the computation of the damage value, because of different damage meanings both express, so we can not directly compare, but it is consistent to judge whether the frame member is damaged and the damage degree. For example, for the frame member $\mathrm{AB}$, comparing with other pole members, the damage values computed by two sorts of method are maximum values, and for the frame member DK, the former computation results are 0.06 and 0.09 , and the later computation results are 0.09 , which indicates the frame member DK is not damaged basically.

\section{Conclusions}

From above analysis, we can obtain two conclusions.

First, because the method in the article can be used to directly analyze the damage value of the two ends of the frame member, it can be extensively applied in diagnosing the damages because of earthquake or other sudden loads or evaluation the surplus credibility.

Second, the mature interface of the finite unit commercial software can be utilized to realize the mechanical model in the method and develop applied program conveniently.

\section{References}

Anil K. Chopra. (2005). Dynamics of structures (Theory and applications to Earthquake Engineering). 2005.5.

Cipollina, A. López-Inojosa and J. Flórez-López. (1995). A simplified damage mechanics approach to nonlinear analysis of frames. Comput. Struct. No.546. P.1113-1126.

Fabio F. Taucer. Earico Spacone. (1991). A fiber beam-column element for seismic response analysis of reinforced concrete structures. Earthquake engineering research center.

G. Bolzon. (1996). An approximate method for fatigue-life prediction of framed structures. Fatigue Fract. Engng Mater. Struct. P.1481-1491.

Marante and Flórez-López, M.E. Marante and J. Flórez-López. (2002). Model of damage for RC elements subjected to biaxial bending. Engng Struct. No.249. P.1141-1152.

Marante, M.E, Flórez-López.J. (2002). Model of damage for RC elements subjected to biaxial bending. Engng Struct. No.24(9). P.1141-1152. 
Maria Eugenia Marante, Julio Flórez-López. (2003). Three-dimensional analysis of reinforced concrete frames based on lumped damage mechanics. International Journal of Solids and Structures. No.40. P.5109-5123.

Mazza \& Mazza, F. (1998). Modelli di danneggiamento nell'analisi sismica non lineare di strutture intelaiate in C.A. (in Italian). Tesis, Università degli Studi della Calabria Cosenza, Italy.

Perera, A. Carnicero, E. Alarcón and S. Gómez. (2000). A fatigue damage model for seismic response of RC structures. Comput. Struct. No.78. P.293-302.

Xue, Weichen \& Zhang, Zhitie. (1999). Nonlinear full range analysis methods of steel concrete structures and its application. Chinese Journal of Computational Mechanics. No.16(3). P.334-342.

Zhu Bolong \& Yu Andong. (1991). Nonlinear full-range analysis of R.C. frames. Journal of tongji university. No.83(3). P.21-30.

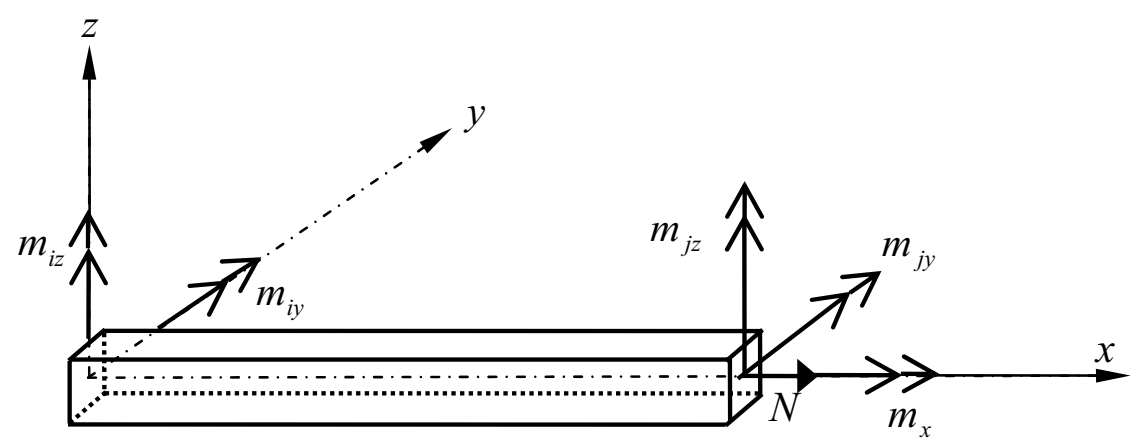

Figure 1. Generalized Stresses in a Frame Member

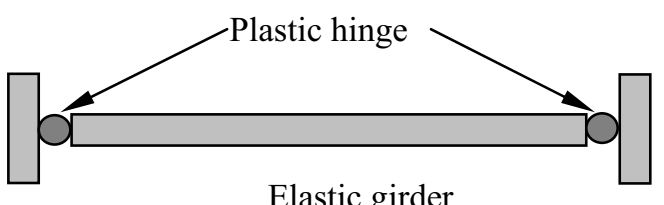

Figure 2. Inelastic Hinges in Frame Member
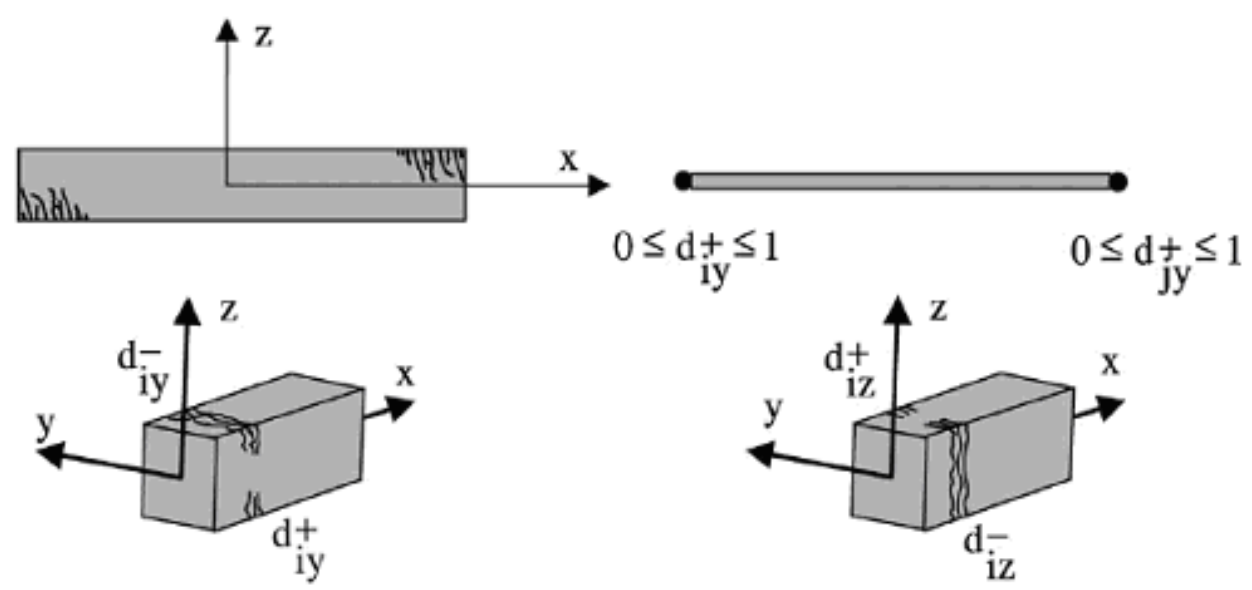

Figure 3. Cracking in a RC Frame Member via Damage Variables 


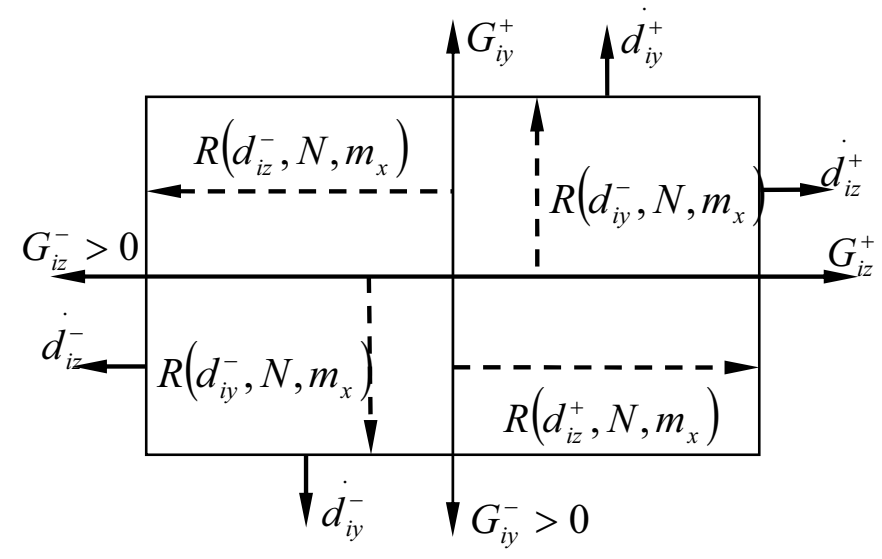

Figure 4. Damage Criterion in the Energy Release Rate Space

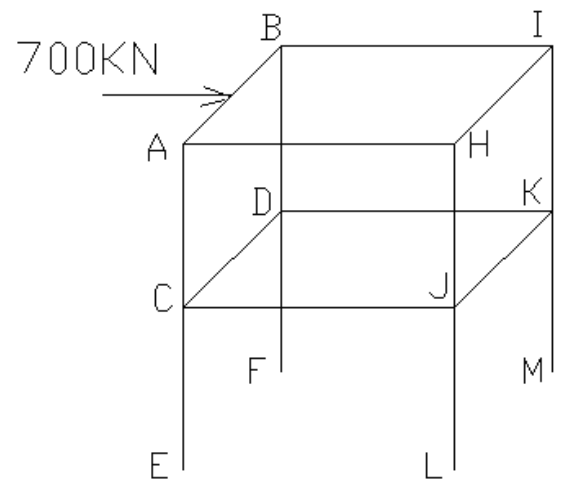

Figure 5. Finite Element Model of the Frame Structure

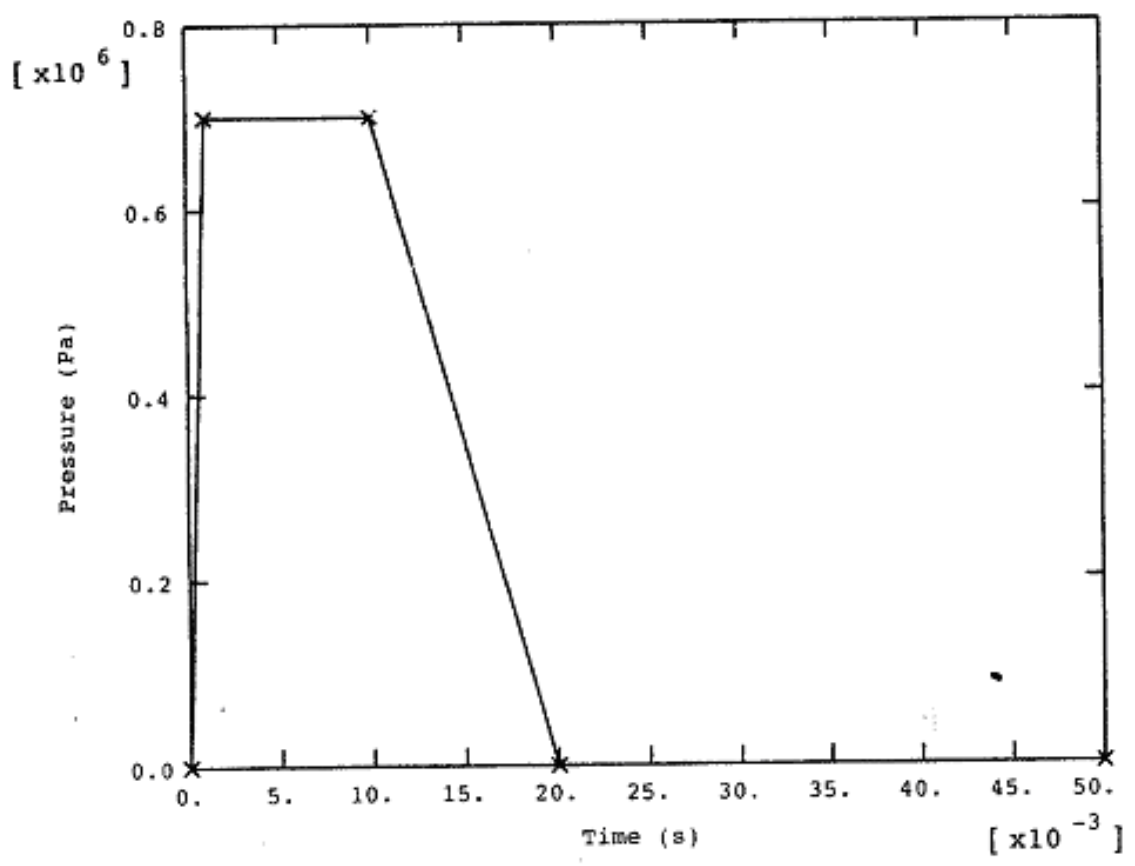

Figure 6. The Impulsive Load as the Function of Time 

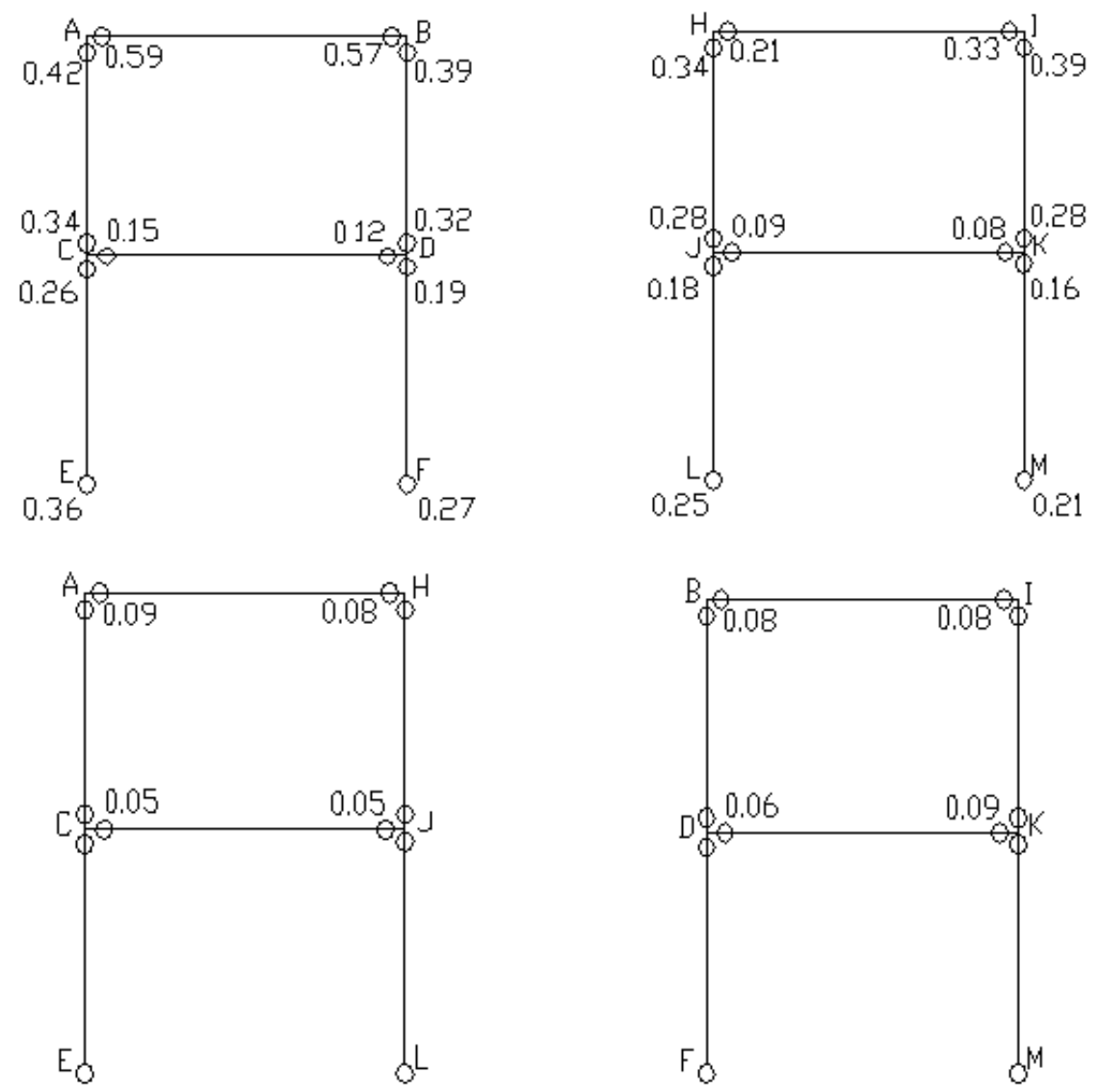

Figure 7. Result of Lumped Damage Mechanics

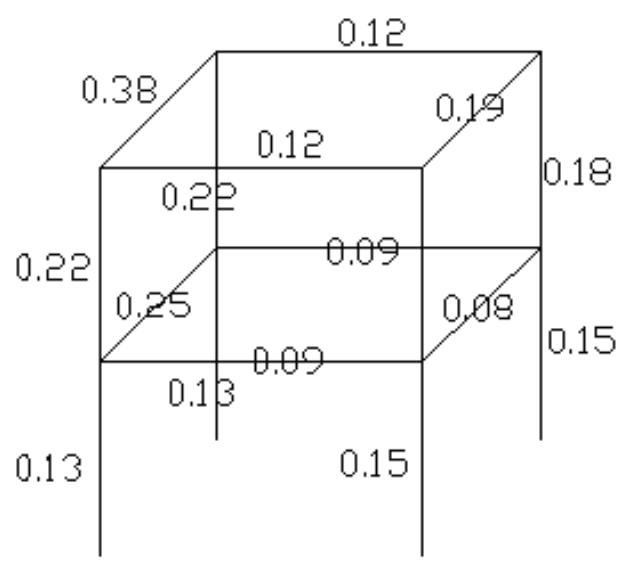

Figure 8. Result of EXPLICIT 